\begin{tabular}{ll} 
Abstract PTH-142 Figure 1 & \\
\hline Diagnosis & Number of patients \\
\hline Adenocarcinoma & 4 \\
Gastrointestinal stromal tumour & 4 \\
Carcinoid & 3 \\
Lymphoma (mantle and follicular) & 3 \\
Metastatic (melanoma and transitional cell bladder) & 3 \\
Glomus tumour & 1 \\
\hline
\end{tabular}

Patient demographics, clinical presentation, findings at DBE and subsequent follow up data were recorded. The majority of patients had a number of investigations, including radiology, prior to DBE without a firm diagnosis. $83.3 \%$ had CE prior to DBE. SPSS V18 was used to analyse the data.

Results A total of 358 DBE procedures were carried out during the stated time period. Of these procedures, 18 (5.0\%) were for the indication of suspected small bowel tumour. The majority $(57.1 \%)$ of these patients were female and the average age at the time of diagnosis was 58 years $(\mathrm{SD} \pm 12 \mathrm{y})$. Indications for performing DBE included iron deficiency anaemia 44.4\%, overt bleeding $27.8 \%$, abnormal radiology $22.2 \%$ and abdominal pain $5.6 \%$. Of those who had prior CE, CE was positive in $93.3 \%$ $(14 / 15)$ of patients with the presence of a mass lesion $(50 \%)$, stricture or ulceration (35.7\%) or the presence of blood alone $(14.3 \%)$. Anterograde DBE was performed in $88.9 \%$ whilst the remaining number had DBE via the retrograde route. Tumour was successfully identified at DBE in $78 \%(\mathrm{n}=14)$ of patients, where tattoos were placed and histology obtained. Of the 4 patients where DBE failed to reach the lesion, the diagnosis was confirmed by laparotomy in 3 patients and intra-operative endoscopy in 1 patient. The final histological diagnosis of tumours identified is shown in Figure 1, UK. $61.1 \%$ tumours were located in the jejunum, $27.8 \%$ in the ileum and $11.1 \%$ in the distal duodenum. DBE influenced ongoing management in all patients that achieved a diagnosis from the procedure.

Conclusion DBE plays a valuable role in the investigation pathway of patients with suspected small bowel tumours. The step wise approach of CE followed by DBE allows efficient use of small bowel resources.

Disclosure of Interest None Declared.

\section{PTH-143 CHARACTERISTICS AND OUTCOMES OF ACUTE INFECTIVE DIARRHOEA- A LARGE TEACHING HOSPITAL COHORT}

'S Mumtaz*, 'S Kelly, 'UA Ahmad, 'S Everett. 'Gastroenterology, Leeds Teaching Hospitals, Leeds, UK; ${ }^{2}$ Acute Medicine, Doncaster Royal Infirmary, Doncaster, UK

\subsection{6/gutjpl-2014-307263.589}

Introduction The clinical syndrome associated with infective diarrhoea is well known, however there are few studies which have rigorously analysed the characteristics in history, examination and investigation in a single cohort of patients.

Methods This was a retrospective cohort study of patients admitted to the gastroenterology department of a single tertiary care teaching hospital with a culture positive episode of bacterial infective diarrhoea, excluding Clostridium difficile. Data was collected from clinical notes of patients presenting over an 8 year period (2004 - 2012) with a follow up period of 12 months from the date of admission.

Results 103 patients were included in the study; 59 (57\%) males, mean age 43 (17-101). Organisms cultured were;
Campylobacter 84, Salmonella 13, Shigella 2, E coli 4. Mean duration of symptoms was 5.7 days (1-14) while patient gave history of; abdominal pain $88 \%$ (91), per rectal (PR) bleeding $52 \%$ (53), vomiting 43\% (44), weight loss 22\% (23), food history $27 \%(28)$, and recent travel $8.5 \%$ (9). 13 patients $(12 \%)$ had pre-existing inflammatory bowel disease (IBD) and 35 patients (34\%) were on a PPI. Examination revealed; fever (>37.5) 35\% (35), tachycardia 27\% (28) and hypotension in 4\% (5) patients. Blood test showed; elevated CRP $(>10)$ in $98 \%$ patients [median 81 (IQR 99)], high white cell count $(>11)$ in 12\% [median 7.8 (IQR 3.7)] and a raised platelet count $(>400)$ in 5\% patients [median 241 (IQR 92)]. Steroids were given to $24(23 \%)$ patients, 9 had pre-existing IBD, mean duration 3 days where as 30 patients (29\%) were given a course of antibiotics after the culture results were known. Mean length of stay was 5 days $(1-30)$; there were 3 in-patient deaths and no surgical interventions. At 12 months 1 patient was diagnosed with IBD but there were no deaths or episodes of re-infection.

Conclusion Acute onset diarrhoeal symptoms associated with abdominal pain, vomiting, or pr bleed and elevated inflammatory markers especially CRP should raise the suspicion of an infective aetiology even if the patient has pre-existing IBD. One in three patients was noted to have pyrexia but contrary to popular belief a history of travelling or food was not a common occurrence.

Disclosure of Interest None Declared.

\section{PTH-144 NATIONWIDE STUDY OF EMERGENCY ADMISSIONS TO ACUTE HOSPITALS IN ENGLAND FOR ANOREXIA NERVOSA: IMPLICATIONS FOR MARSIPAN}

${ }^{1} \mathrm{M}$ Shawihdi ${ }^{*},{ }^{2} \mathrm{E}$ Thompson, ${ }^{3} \mathrm{~S}$ Sharma, ${ }^{4} \mathrm{~S}$ Lal, ${ }^{2} \mathrm{M}$ Pearson, ${ }^{1} \mathrm{~K}$ Bodger. ${ }^{1}$ Department of Gastroenterology, Institute of Translational Medicine, University of Liverpool, Liverpool, UK; ${ }^{2}$ Aintree Health Outcomes Partnership, University of Liverpool, Liverpool, UK; ${ }^{3}$ Priory Eating Disorders Services, The Priory Hospital Cheadle Royal, Cheadle, UK; ${ }^{4}$ Gastroenterology and Intestinal Failure Unit, Salford Royal NHS Foundation Trust, Salford, UK

\subsection{6/gutjnl-2014-307263.590}

Introduction Patients with anorexia nervosa (AN) develop significant physical complications leading to emergency admission (EmAd) to acute hospitals. There are few data on national burden, institutional case volume, frequency, nature or outcomes of EmAds for this rare, complex condition. The need for joint management of AN between medical and psychiatric teams has been highlighted ${ }^{1}$. We aimed to define characteristics of adult patients with AN admitted as emergencies to acute hospitals in England. Methods A 2-year download of data for English acute hospitals (Hospital Episode Statistics) was obtained, linked to death registry. We extracted all EmAds in medical or surgical specialties containing ICD-10 codes for AN. Adult patients with a first (index) admission between Oct 07 and Sept 08 were selected (1year incident cohort). Admissions in 6 months before or after index admission were extracted and ordered chronologically. Demographics and diagnosis codes for each admission were reviewed independently by two gastroenterologists $(\mathrm{SL}, \mathrm{KB})$ and a psychiatrist (SS), selecting only cases where 2 of 3 reviewers judged the coding sequence consistent with EmAd for AN. The index EmAd was classified according to primary diagnosis.

Results 549 AN patients were admitted to 132 Trusts in England during the year. Mean age [sd]: 30 [11] yrs; Female: 95.4\%; $\geq 1$ Charlson co-morbidities: $11.3 \%$. Primary diagnosis: AN, 33.5\%; Complication of AN or a GI symptom, 39.2\%; Poisoning or Self Harm, 17.5\%; Alcohol-related, 2.4\%; Miscellaneous diagnoses, 